\title{
A Spectrum Sensing Algorithm Based on Maximum Entropy Spectral Estimation
}

\author{
Jiangzhou Wang \\ Xi'an University of Posts and Telecommunications, China \\ wangjiangzhou@163.com
}

Keywords: cognitive radio; spectrum sensing; PAAR; Maximum Entropy Spectral Estimation.

\begin{abstract}
A spectrum sensing algorithm, named as MESE algorithm, based on maximum entropy spectral estimation was constructed in this papers. Frequency domain analysis was introduced into the spectrum sensing, peak to average power spectrum amplitude ratio (PAAR) was taken as detection statistics, Detection threshold obtained from the probability density distribution of the detection statistic by the method of Monte Carlo. The simulation results show that the detection performance of MESE algorithm was much better than energy detection algorithm.
\end{abstract}

\section{Introduction}

With the rapid development of wireless communication technology and the rapid growth of wireless communication services, the demand for spectrum resources was growing fast, the lack of spectrum resources has become one of major bottleneck in the development of wireless communications[1]. Many scarce hotspots spectrum resources assumed great traffic, and the utilization of a large number of authorized spectrum resource was very low, idled in most of the time, and cognitive radio was invented to solve this contradiction to improve spectrum efficiency [2].

Spectrum sensing was the key technology of cognitive radio systems, the research for cognitive radio spectrum sensing has important implications. Many studies have been carried out on the spectrum sensing at home and abroad. Energy detection algorithm was a classic spectrum sensing algorithm, but the performance of which was easily affected by the uncertainty of noise. In order to improve the quality of spectrum sensing, a spectrum sensing algorithm based on maximum entropy spectral estimation was constructed.

\section{System Model and Performance parameters of Spectrum Sensing Algorithm}

\subsection{System Model}

The weak signal detection was the basis of spectrum sensing, spectrum sensing system should be able to detect this signal if the authorized user signals appears in a frequency band. The essence was a binary hypothesis testing issue, hypothesis testing model was established as shown in Eq. 1

$$
\begin{aligned}
& H_{1}: \quad x(k)=h(k) * s(k)+n(k) \\
& H_{0}: \quad x(k)=n(k)
\end{aligned}
$$

$\mathrm{x}(\mathrm{k})$ represents the reception signal, $\mathrm{s}(\mathrm{k})$ represents the transmission signal, $\mathrm{h}(\mathrm{k})$ represents the channel response, $\mathrm{n}(\mathrm{k})$ represents additive white Gaussian noise, * represents convolution.

$\mathrm{H}_{1}$ represents the frequency band to be used, $\mathrm{H}_{0}$ represents idle band, Herein $\mathrm{h}(\mathrm{k})=1$ is assumed, the decision process can be expressed in Eq. 2

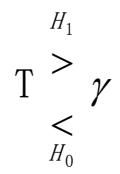


T represents detection statistic extracted from the received signal, $\gamma$ represents the decision threshold. The decision was $\mathrm{H}_{0}$ when $\mathrm{T}<\gamma$, and the decision was $\mathrm{H}_{1}$ when another case.

\subsection{Performance Parameters}

There were four possible analyzing results of binary hypothesis test, they were[3]:

1) Detection probability, abbreviated as $\mathrm{P}_{\mathrm{d}}$, represents $\operatorname{Prob}\left(\mathrm{H}_{1} / \mathrm{H}_{1}\right)$;

2) Missed detection probability, abbreviated as $\mathrm{P}_{\mathrm{m}}$, represents $\operatorname{Prob}\left(\mathrm{H}_{0} / \mathrm{H}_{1}\right)$;

3) False alarm probability, abbreviated as $\mathrm{P}_{\mathrm{f}}$, represents $\operatorname{Prob}\left(\mathrm{H}_{1} / \mathrm{H}_{0}\right)$;

4) The last case was $\operatorname{Prob}\left(\mathrm{H}_{0} / \mathrm{H}_{0}\right)$;

\section{MESE Detection Algorithm}

The maximum entropy spectral estimation has strong noise immunity, therefore spectrum sensing algorithm based on MESE was designed to improve the performance of spectrum sensing[4].

\subsection{The choice of test statistics}

The spectral estimation was an important part of MESE algorithm, which was estimated, respectively, for pure noise signals and user signals under the conditions $\sigma^{2}{ }_{n}=1$ and $\alpha=1$ [7], as was shown in Fig. 1 and Fig. 2.

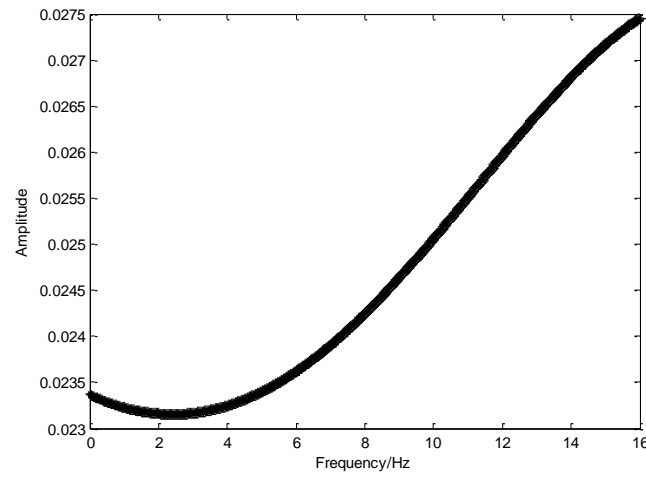

Fig. 1. MESE of noise, $\mathrm{H}_{0}$

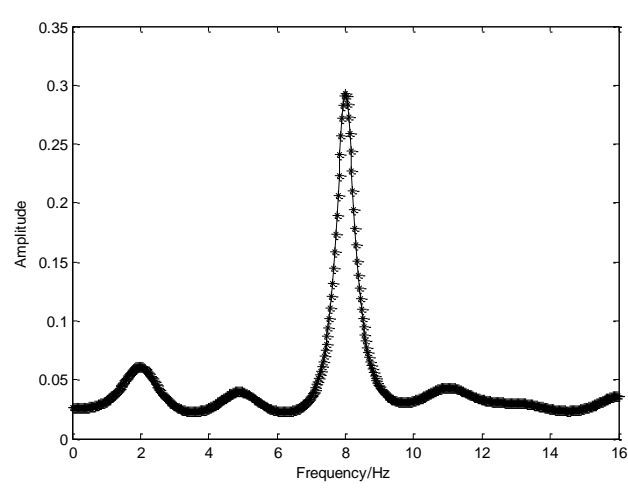

Fig. 2. MESE of user signal, $\mathrm{H}_{1} \mathrm{SNR}=-5 \mathrm{~dB}$

It could be found that there was great distinction of the amplitude of power spectral density between the conditions of $\mathrm{H}_{1}$ and $\mathrm{H}_{0}$. The Peak-to-Average Amplitude Ratio (PAAR) of power spectral density of received signal was taken as the detection statistic, in this paper, the $\mathrm{Q}$ is used to represent PAAR, as shown in Eq. 3

$$
Q=\frac{S_{\max }}{S_{\text {mean }}}
$$

$\mathrm{S}_{\max }$ represents the peak of the power spectrum, $\mathrm{S}_{\text {mean }}$ represents the average amplitude of power spectrum.

\subsection{Algorithm steps}

The steps of MESE algorithm were shown below:

(1) Generating QPSK modulation(detection sequence length M was set be 3200 in followed simulations ), raised cosine pulse shaping and noise signals by means of simulation, and can control uncertainty of noise and SNR;

(2) Sampling data, and completed the maximum entropy spectral estimation;

(3) Selected the highest peaks in the generated power spectrum and add window, Obtained the maximum and the $S_{\text {mean }}$;

(4) calculated Detection statistic Q;

(5) Depending on the probability of false alarm $P_{f}$ requirements,

(6) Determined decision threshold by Monte Carlo simulation;

(7) Decided the property of the received signal. 


\section{Performance Simulation of MESE Detection Algorithm}

The performance of MESE detection algorithm was simulated in this section, which was also compared with the ED algorithm, and the influence of the noise uncertainty was considered deliberately.

The anti-noise performance was an important indicator in measure a spectrum sensing algorithms, the main parameters of noise including noise variance $\sigma_{n}^{2}$ and noise Uncertainty, represented by $\alpha$. Assuming the noise uncertainty was $\alpha \mathrm{dB}, \beta=10^{\alpha / 10}$, the actual noise energy $\sigma^{2}$ fluctuated within the range of $\left[\sigma_{\text {est }}^{2} \beta \cdot \sigma_{\text {est }}^{2}\right], \sigma_{\text {est }}^{2}$ represents estimates of the noise energy[5]. The noise signal variance was set as 1 or 10 and noise signal uncertainty was set as 0 or 1 usually.

Unlimited data could only be approximate substituted because of the limited samples data, and this produced certain bias between the detection statistic Q and the theoretical value, as was shown in Fig. 3. With the increasing of samples points, simulation value approach the theoretical value gradually. It also be summarized that mean distribution of detection statistic was almost independent with noise variance and noise uncertainty in the four cases listed in Fig. 3.

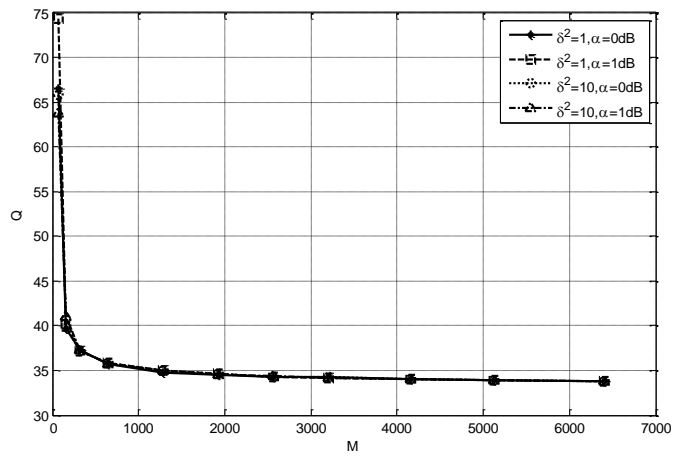

Fig. 3. Q value and sampling point

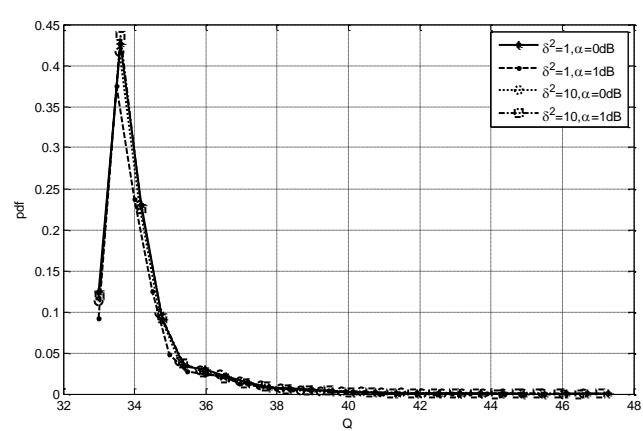

Fig. 4. Probability density distribution of $\mathrm{Q}$ value, $\mathrm{H}_{0}$

It was an important indicator of an algorithm that the $\mathrm{P}_{\mathrm{d}}$ can be achieved under certain conditions of the $\mathrm{P}_{\mathrm{f}}$, and the detection threshold was the key to get the $\mathrm{P}_{\mathrm{d}}$.

As shown in Fig. 4, the probability density distribution of detection statistic was obtained by means of ten thousand times Monte Carlo simulation. The curves in the graph represent the probability density distribution of detection statistics $Q$ under the conditions of $\alpha=0 \mathrm{~dB}, \alpha=1 \mathrm{~dB}, \sigma^{2}{ }_{n}=1$ and $\sigma^{2}{ }_{n}=10$ respectively. It was shown that detection Statistics Q set by this algorithm almost didn't affected by noise variance and uncertainty, and this was consistent with the results in Figure 3.

The noise variance and the noise uncertainty were constant, they were set as $\sigma_{n}^{2}=1, \alpha=1$, in the condition of $\mathrm{H}_{0}$ and $\mathrm{H}_{1}$, SNR were set separately as $\mathrm{SNR}=-10 \mathrm{~dB}$ and $\mathrm{SNR}=-15 \mathrm{~dB}$. The three cases probability density distribution of detection Statistics Q were shown in Fig. 5. It was shown that probability density distribution in the condition $\mathrm{H}_{0}$ hadn't any overlap with the probability density distribution in the condition of $\mathrm{SNR}=-10 \mathrm{~dB}$, and the $\mathrm{P}_{\mathrm{d}}$ could up to $100 \%$ in this case.

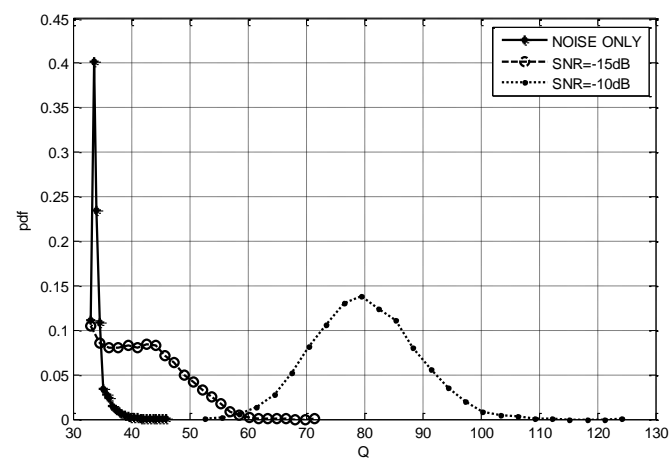

Fig. 5. Probability density distribution of $\mathrm{Q}, \mathrm{H} 0$ and $\mathrm{H} 1$

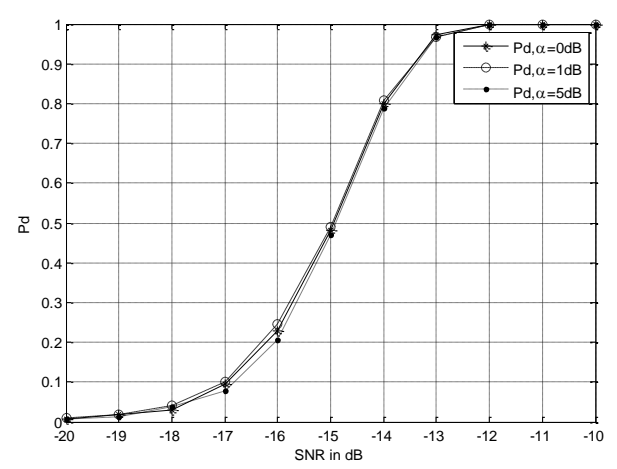

Fig. 6. $\mathrm{P}_{\mathrm{d}}$ of MESE algorithm, $\mathrm{P}_{\mathrm{f}}=10^{-4}$

The $P_{d}$ under different $S N R$ were obtained in the condition of $P_{f}$ was set as $10^{-4}$, as shown in Fig. 6 . It was summarize that $\mathrm{P}_{\mathrm{d}}$ reached $100 \%$ when the $\mathrm{SNR}$ was greater than $-12 \mathrm{~dB}$. It was also can be 
summarize that detection performance of MESE algorithm was not affected by noise uncertainty even when $\alpha=5 \mathrm{~dB}$.

The performance of MESE[6] algorithm and ED algorithm were compared in the condition of $\mathrm{P}_{\mathrm{f}}=$ $10^{-4}$, and the result shown in Fig. 7.

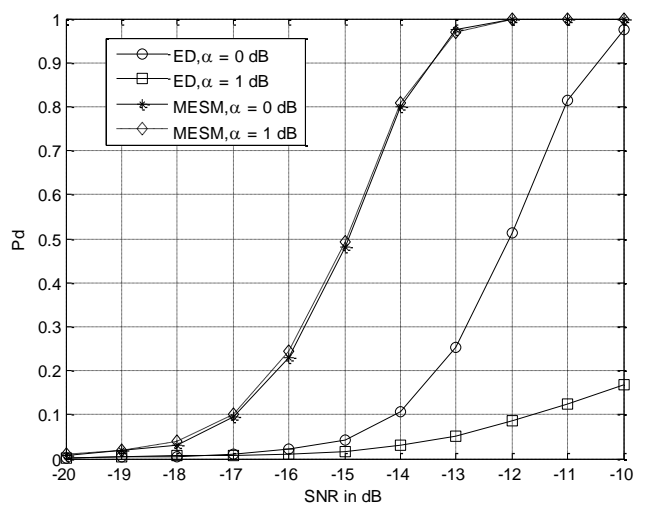

Fig. 7 The $\mathrm{P}_{\mathrm{d}}$ Comparison between MESE algorithm and ED algorithm, $\mathrm{P}_{\mathrm{f}}=10^{-4}$

(1)The Pd of MESE algorithm was up to $97 \%$, The Pd of ED algorithm was only about $26 \%$ in the condition of SNR $=-13 \mathrm{~dB}$ and $\alpha=0 \mathrm{~dB}$. Obviously, The Pd of MESE algorithm was much better than ED algorithm's in low SNR.

(2)The Pd of ED algorithm Reduced to $17 \%$ from the $97 \%$ when the SNR was set at SNR $=-10 \mathrm{~dB}$ and the uncertainty noise increased to $\alpha=1 \mathrm{~dB}$, but the Pd of MESE algorithm almost unchanged in the same case. The MESE algorithm was almost not affected by the uncertainty of noise visibly

\section{Conclusions}

A spectrum sensing algorithm, named MESE detection algorithm, based on maximum entropy spectral estimation was constructed in this paper, frequency domain analysis was introduced into the algorithm, peak to average power spectrum amplitude ratio (PAAR) was taken as detection statistics, detection threshold obtained from the probability density distribution of the detection statistic by the method of Monte Carlo, Finally, achieved the judgment of signal properties.

The simulation results show that the MESE algorithm overcomes the shortcomings that ED algorithm affected by noise uncertainty excessively, and can still achieve high $\mathrm{P}_{\mathrm{d}}$ under the requirements of $P_{f}=10^{-4}$ and low SNR, the detection performance of MESE algorithm was much better than ED algorithm's visibly.

\section{References}

[1] Wang Jiangzhou. Measurements and Analysis of Spectrum Occupancy. JOURNAL OF UNIVERSITY OF POSTS AND TELECOMMUNICATIONS[J].2014.09. 10-11

[2] Mitola J, Maquire G Q. Cognitive Radio: Making Software Radios More Personal. IEEE Personal Communications [J]. 1999, 6(4):13-18。

[3] Zhao Shujie, Zhao Jianxun. Signal Detection and Estimation Theory. [M]. Beijing: Tsinghua University Press, 2005: 68-69。

[4] Zhao Chenglin. Study on spectrum sensing method based on Maximum Entropy Spectral Estimation[J]. China ACADEMY OF ELECTRONICS. 2010.10. 508-509.

[5] Wang Yingxi. Spectrum sensing in cognitive radio system by use of antenna array[D].2010. $16-18$.

[6] Lu Guanghua, Peng Xueyu. Random Signal Processing[M].Xi'an:Xidian University Press.2002. 104-109.

[7] Zhang Linghua, Zheng Baoyu. Random Signal Processing[M]. Beijing: Tsinghua University Press, 2003: 100. 